\title{
Sexismo y formación inicial del profesorado
}

\author{
Raúl Carretero Bermejo \\ Universidad de Castilla-La Mancha. España. \\ info@raulcarreterobermejo.com
}

Alberto Nolasco Hernández

Universidad de Zaragoza. España.

anolasco@unizar.es

Recibido: 19/4/2016

Aceptado: 21/9/2016

Publicado: 26/10/2017

\section{Resumen}

Este trabajo, enmarcado dentro de un proyecto de investigación más amplio, tiene por objetivo fundamental conocer las creencias y las actitudes sexistas de los futuros docentes como responsables de la educación y como trasmisores ideológicos en el día de mañana.

En el análisis que exponemos a continuación, la población de alumnos de Magisterio presentó creencias sexistas en más de un $75 \%$ de la muestra estudiada, lo que significa que más de un $75 \%$ de futuros docentes está en disposición de trasmitir esta ideología frente a las diversas propuestas legislativas que persiguen la igualdad. Las personas objeto de estudio han mostrado actitudes sexistas hostiles y también benévolas. Los resultados encontrados justifican la necesidad de programar acciones formativas y educativas concretas y explícitas para el colectivo de futuros docentes, con el fin de cambiar estas creencias y detener así la perpetuación y la trasmisión ideológica del sexismo. Por tanto, parece necesaria la inclusión de formación específica en género y sexismo, así como la programación y el diseño de los planes de estudio desde la perspectiva de género. Estos resultados también muestran la necesidad de conocer las actitudes sexistas, o no sexistas, de los enseñantes en activo, dado que, si se confirman estos resultados entre la población docente, las necesidades de formación también se extenderían al grupo de profesores que ejercen en la actualidad.

Palabras clave: sexismo; actitud; educación; estudiantes; prejuicio; estereotipo

Resum. Sexisme i formació inicial del professorat

El present treball de recerca pretén conèixer les creences sexistes de la població d'estudiants de Magisteri, perquè les facultats d'educació són les que formen i treballen amb les persones responsables de l'educació i la formació dels nostres menors. L'escola, com a institució, i els docents, com a màxims representants d'aquesta institució, són uns responsables fonamentals en els processos de transmissió cultural i ideològica, en aquest cas, la referida a l'actitud sexista.

En l'anàlisi que exposem a continuació, la població d'alumnes de Magisteri presenta creences sexistes en més d'un $75 \%$ de la mostra estudiada, la qual cosa significa que més d'un $75 \%$ de futurs docents transmetrà aquesta ideologia enfront de les diverses propostes legislatives que persegueixen la igualtat. La població objecte d'estudi presenta actituds 
sexistes hostils i també benèvoles. Els resultats trobats justifiquen la necessitat de programar accions formatives i educatives concretes i explícites per al col-lectiu de futurs docents, amb la finalitat de canviar aquestes creences i, d'aquesta manera, detenir la perpetuació i la transmissió ideològica del sexisme. Per tant, sembla necessari incloure formació específica en gènere i sexisme, a més de programar i dissenyar els plans d'estudi des de la perspectiva de gènere. Aquests resultats també mostren la necessitat de conèixer les actituds sexistes, o no sexistes, dels ensenyants en actiu, atès que, si es confirmen aquests resultats entre la població docent, les necessitats de formació també s'estendrien al grup de professors que exerceixen actualment.

Paraules clau: sexisme; actitud; educació; estudiants; prejudici; estereotip

\section{Abstract. Sexism and initial teacher training}

This paper, which is framed within a broader research project, aims to determine the sexist beliefs and attitudes of future teachers as educators and ideological transmitters. More than $75 \%$ of the sample of student teachers analyzed exhibits sexist beliefs, thus suggesting that more than $75 \%$ of future teachers will transmit this sexist ideology in contrast to legislative proposals that pursue equality. The study population exhibits hostile and benevolent sexist attitudes. The results indicate the need to develop specific training and educational activities for future teachers in order to change these beliefs and impede the perpetuation and ideological transmission of sexism. To this end, specific training in gender and sexism and the programming and design of curricula from a gender perspective is necessary. Moreover, it is necessary to determine whether in-service teachers exhibit sexist attitudes. If such attitudes are confirmed, gender-equality training should be extended to include in-service teachers.

Keywords: sexism; attitude; education; student teacher; bias; stereotype

\begin{aligned} & \multicolumn{2}{c}{ Sumario } \\ & 1. Introducción 6. Conclusiones \\ & 2. Marco teórico: sexismo 7. Limitaciones de la presente \\ & 3. Metodología investigación \\ & 4. Resultados 8. Líneas de trabajo abiertas \\ & 5. Análisis y discusión de los resultados Referencias bibliográficas \end{aligned}

\section{Introducción}

En la realización de una práctica con estudiantes de Magisterio, estos mostraban, en su mayoría, creencias y actitudes sexistas de manera abierta. Nos preguntamos si esta observación era cierta o si se trataba solo de una percepción, por lo que decidimos pasar el inventario de sexismo ambivalente (ASI) (Expósito, Moya y Glick, 1998). Al observar los resultados obtenidos mediante la prueba, comprobamos que, efectivamente, nuestros estudiantes obtenían puntuaciones que indicaban la presencia de actitudes y de creencias sexistas en más de la mitad de los miembros del grupo. Estos resultados nos llevaron a plan- 
tearnos varias preguntas. La primera trataba acerca de si estos se repetirían si la muestra fuera representativa del colectivo de estudiantes de Magisterio de Castilla-La Mancha o si, por el contrario, constituía solo un dato concreto en un grupo concreto. La segunda, si los resultados se repetían, deberíamos plantearnos si, con esas hipotéticas creencias y actitudes sexistas, los futuros profesores podrían desarrollar una práctica pedagógica desde y para la igualdad. Y la tercera, si, desde el sistema educativo, se estaba actuando desde el punto de vista de la programación para trabajar con estas hipotéticas creencias y actitudes sexistas o si la actuación únicamente se limitaba a la norma.

Los términos como coeducación, educación no sexista o educación para la igualdad no son nuevos. Parece, sin embargo, que los esfuerzos relativos a este supuesto trabajo a favor de la igualdad han quedado reflejados en los diversos y continuos marcos legislativos de manera más o menos explícita, pero no en la programación de medidas para tratar el sexismo con los futuros docentes, por lo que se ha dado por hecho que la simple regulación implicaría un cambio en las ideologías de los profesores objeto de la regulación. En concreto, nuestras dos últimas normas relativas a la enseñanza, tanto la Ley Orgánica 2/2006 de educación (LOE) como la Ley Orgánica 8/2013 para la mejora de la calidad educativa (LOMCE), señalan y mencionan de manera explícita objetivos y contenidos relacionados con la igualdad entre hombres y mujeres, la no discriminación por razón de sexo o género y la lucha contra la violencia de género.

Quedan claras, por tanto, las intenciones legisladoras, pero no está tan claro si los futuros docentes están en disposición de implementar estas normas y desarrollar una educación efectivamente no sexista. Esta declaración normativa no solo no se ha trasladado al diseño de los planes de estudio de las facultades de educación en forma de objetivos y materias en relación con la capacitación profesional para desarrollar estos textos legislativos, sino que tampoco se ha desarrollado otra vía de formación en forma de contenidos de los llamados de libre configuración o en los cursos de formación que sumarán créditos en el aprendizaje de estos docentes. Ni tan siquiera existe una oferta formativa abierta o general a la que los profesores también puedan acceder en relación con los contenidos relacionados con el sexismo, la igualdad o la perspectiva de género.

En primer lugar, cabe preguntarse, por tanto, acerca de la formación necesaria del cuerpo docente para la consecución y el trabajo de estas metas, así como acerca del modo de articular esta formación incluida en planes de estudios, en la formación complementaria y en la formación continua del profesorado o si es mejor dejarlo en manos de cada una de las personas que dedican su vida a la enseñanza (hasta el momento, esta es la opción elegida desde la Administración). En segundo lugar, pero no menos importante, cabe preguntarse también acerca de las creencias y de las actitudes desde las que es posible realizar esta labor por y para la igualdad y sobre cuáles son las creencias y las actitudes de las personas que ejercerán la docencia en el futuro, así como si estas creencias son compatibles con las exigencias legales o no. Podríamos preguntarnos también acerca de las consecuencias de una educación desarro- 
llada e implementada desde el sexismo, una escuela con enseñantes que mostraran actitudes sexistas y un modelo educativo legislado, evaluado y creado también desde la existencia de actitudes sexistas, en el caso de que estas estuvieran en dichos contextos. También surgen preguntas relativas a qué leyes estamos construyendo para erradicar el sexismo si estas se desarrollan desde el propio sexismo.

Se ha escrito y se ha estudiado acerca de la necesidad de incluir, en los planes de estudio, contenidos sobre coeducación y educación no sexista. También existe literatura científica sobre la influencia del sexismo en la educación, el sexismo en las aulas y las estrategias metodológicas para trabajar los contenidos de coeducación, igualdad y no discriminación. Sin embargo, no encontramos información acerca de las creencias y de las actitudes sexistas ni del cuerpo docente ni de las estudiantes y de los estudiantes que aspiran a formar parte de él. Para encontrar información referida al sexismo en función del sexo y poder comparar nuestros resultados, hemos tenido que acceder a investigaciones desarrolladas en otras poblaciones. Según los resultados obtenidos en trabajos anteriores acerca de la relación del sexo con las creencias sexistas, las mujeres presentan puntuaciones más altas en sexismo benévolo que en sexismo hostil (Rodríguez, Lameiras, Carrera y Faílde, 2009; Ayres, Friedman y Leaper, 2009), mientras que los hombres muestran puntuaciones más altas en sexismo hostil (Moya y Expósito, 2001; Lameiras y Rodríguez, 2004). En ambos casos, los hombres puntúan significativamente más alto que las mujeres en las dos dimensiones del sexismo.

Nos planteamos, primero, si esta tendencia se mantiene entre los estudiantes de Magisterio y, segundo, qué implicaciones educativas comportarían estas actitudes sexistas en caso de mantenerse. El sexismo está presente entre las variables de riesgo en situaciones de violencia y acoso escolar (Díaz-Aguado, 2006; Ovejero, Yubero, Larrañaga y Navarro, 2013), está relacionado con la convivencia académica (Díaz-Aguado, Martínez y Martín, 2010) y es una de las principales causas de discriminación hacia las mujeres (Moya, 2004; Garaigordobil y Aliri, 2013), de ahí la importancia de conocer las actitudes de nuestros futuros docentes y, por tanto, el modelo ideológico que trasmitirán.

Esta investigación, enmarcada en el desarrollo de un diseño de investigación sobre sexismo y emoción, surge de la observación de las medidas y de las puntuaciones obtenidas en sexismo por nuestra muestra - formada por estudiantes de magisterio de Castilla-La Mancha- y tiene como objetivo fundamental determinar y evaluar las actitudes sexistas de las estudiantes y los estudiantes de Magisterio, puesto que son el futuro inmediato de la educación y, por lo tanto, los que más adelante trasmitirán ideología y valores culturales, en este caso relacionados con el sexismo. No nos engañemos, la norma no es suficiente, es necesario también que se realice un cambio ideológico, actitudinal y de sistema de creencias, no solo por parte de los profesionales, sino también de la institución.

La existencia de esta actitud entre los futuros docentes justificaría por sí misma la necesidad de implementar acciones formativas relacionadas con el género, la perspectiva de género y el sexismo, no solo a nivel técnico y teórico, 
sino, sobre todo, a nivel de modificación y de eliminación de estas actitudes sexistas, si realmente queremos implementar y desarrollar lo que sí se legisla. Derivadas de este objetivo, surgen nuestras hipótesis de trabajo: más del 51\% de los estudiantes y las estudiantes de Magisterio presentan creencias sexistas hostiles y benévolas. Además, pensamos que los alumnos de Magisterio puntúan más alto en sexismo que las alumnas de Magisterio; los hombres puntúan significativamente más alto en sexismo hostil que en sexismo benévolo, y las mujeres puntúan más alto en sexismo benévolo que en sexismo hostil. Este segundo grupo de hipótesis es importante, dado el número de hombres y de mujeres matriculados en este grado, lo que, por lo tanto, constituye un indicador de la forma en la que se trasmitirá el sexismo, en caso de que se confirmen nuestras hipótesis de trabajo.

\section{Marco teórico: sexismo}

Tradicionalmente, el sexismo ha sido considerado una actitud de prejuicio hacia las mujeres y una de las principales actitudes que mantienen las desigualdades entre sexos (Moya, 2004).

El sexismo hace referencia a un conjunto de creencias que aparecen como resultado de la desigual representación social de hombres y mujeres. El sexismo se articula conformando una ideología que, vinculada al género, describe y prescribe no solo los roles sociales propios de hombres y mujeres, sino también la forma adecuada de relacionarse los dos sexos. En la actualidad, trabajamos con dos tipos o conceptualizaciones de sexismo: neosexismo, que expone la necesidad de diferenciar las nuevas formas de sexismo, más sutiles y encubiertas, de las formas antiguas (Lameiras, 2004), y sexismo ambivalente, resultante de combinar dos dimensiones sexistas con cargas afectivas antagónicas, donde el sexismo hostil se identifica con el sexismo tradicional y con una valoración subjetiva negativa, y donde el sexismo benévolo se corresponde con la generación de recompensas, formas de protección e idealización de la mujer, con lo que se refuerza su situación de inferioridad, pero con una valoración subjetiva positiva.

Como actitud, el sexismo está constituido por tres componentes (DíazAguado, 2006): el cognitivo, formado por el conjunto de creencias que están en la base de la actitud y a la que dan forma; de ese modo, el sexismo se fundamenta en la creencia errónea de que las diferencias de sexo son la causa de todas las diferencias sociales y psicológicas, por lo que, desde esta visión, esta diferencias son insalvables; el afectivo-valorativo, que asocia los valores de fuer$\mathrm{za}$, poder, control y dureza emocional y uso de la violencia a lo masculino, y la sumisión y la debilidad a lo femenino, y el conductual, que incluye la tendencia a llevar a la práctica las creencias y los valores a través de conductas violentas y de discriminación en el caso de los hombres y de sumisión y culpabilidad en el caso de las mujeres. 


\subsection{Teoría del sexismo ambivalente}

Junto a estas formas hostiles y tradicionales de sexismo, Glick y Fiske (1996, 1999) identifican una nueva, a la que denominan sexismo benévolo, caracterizada por mantener una carga afectiva subjetiva positiva. Esta nueva forma de sexismo benévolo debilita la resistencia de las mujeres, al tiempo que refuerza su posición de inferioridad, y genera recompensas en forma de protección, idealización y afecto para aquellas que aceptan su rol tradicional y satisfacen las necesidades de los hombres, frente al uso del castigo y la violencia fomentados desde el sexismo hostil para aquellas mujeres que no se ajustan al rol tradicional. De esta forma, el sexismo ambivalente es el resultado de la combinación de dos elementos con cargas afectivas antagónicas, consecuencia de las complejas relaciones de aproximación y evitación que caracteriza a las conexiones entre sexos.

Así, desde la teoría del sexismo ambivalente, se considera el sexismo como un prejuicio ambivalente y no una antipatía uniforme hacia las mujeres (Glick y Fiske, 1996). Las relaciones entre los sexos están caracterizadas por una compleja interacción de fuerzas que convergen en determinadas ocasiones y divergen en otras, por lo que, para conocer y comprender el sexismo moderno, es fundamental incluir variables explicativas derivadas de la dimensión relacional que se produce entre los sexos (Rodríguez, Lameiras, Carrera y Faílde, 2009). Esta teoría es la primera en incluir y reconocer la relevancia del análisis de la dimensión relacional en el estudio del sexismo.

Para evitar el conflicto entre las actitudes positivas y negativas hacia las mujeres, el sexismo ambivalente las clasifica en subgrupos, de tal manera que genera un subgrupo de mujeres valorado como positivo y hacia el que actúa de manera benevolente, y un segundo subgrupo de mujeres valorado de manera negativa hacia el que manifiesta conductas hostiles. Esta polarización es una estrategia muy eficaz para evitar conflictos, dado que forma categorías tales como la apariencia, la forma de vestir o los roles sociales que cada mujer desempeña y que guían y determinan su comportamiento individualmente en función de su grupo de pertenencia. Desde esta teoría, se presentan tres subtipos de mujer creados por los hombres: las tradicionales, formado por amas de casa y esposas, madres y cuidadoras; las no tradicionales, formado por mueres que desarrollan su carrera profesional en un espacio público, y, por último, las sexis.

Los hombres sexistas temen a las mujeres no tradicionales, puesto que amenazan su poder, y temen a las sexis, al entender que su poder de seducción y su interés por el sexo les puede hacer también perder el poder. Las mujeres sexis y las no tradicionales son el blanco de las conductas más hostiles. El sexismo hostil se convierte en un castigo para las mujeres no tradicionales, feministas y sexis, mientras que el sexismo benévolo se emplea para recompensar a las mujeres tradicionales. Así, los hombres pueden mantener una actitud consistente, puesto que el desprecio o el amor está claramente distribuido y asignado a los diferentes subgrupos (Rudman y Glick, 2001). 
La teoría del sexismo ambivalente tiene su base en variables biológicas y sociales, y se articula en torno a tres dimensiones:

- La primera tiene que ver con la distribución de poder. A esta dimensión, la denominan paternalismo, el cual, en el sexismo hostil, se manifiesta como dominador, mientras que en el sexismo benévolo este paternalismo aparece bajo formas de protección y de actuación protectora hacia las mujeres (lo que lleva implícito el reconocimiento de la inferioridad y de la debilidad de las que necesitan ser protegidas).

- La diferenciación de género es la segunda dimensión. En el sexismo hostil, es de tipo competitivo (la propia del género masculino es más importante y necesaria, con mayor grado de dificultad y complejidad que la propia del género femenino), mientras que, en el sexismo benévolo, es de tipo complementario.

- Por último, la sexualidad aparece como la tercera dimensión encontrada. En el sexismo hostil, las mujeres se muestran como personas sin sexualidad o con una sexualidad muy poderosa, lo que las hace potencialmente peligrosas para los hombres (tradicionalmente, esta creencia ha significado que las mujeres hayan tenido que reprimir su sexualidad). En el otro lado, el sexismo benévolo contempla solo las relaciones heterosexuales, que son consideradas fundamentales para alcanzar la felicidad verdadera.

El significado de sexismo se reconceptualiza continuamente y añade nuevos significados. Sin embargo, parece mantenerse estable el funcionamiento y la existencia de un sexismo hostil y/o tradicional y de un sexismo benévolo.

\section{Metodología}

Para la realización de este trabajo, hemos desarrollado un diseño de investigación cuasiexperimental de corte cuantitativo.

\subsection{Participantes}

Para el desarrollo de la presente investigación, se ha seleccionado una muestra de 1.623 personas, todas ellas estudiantes de Magisterio, repartidas en las cuatro provincias de Castilla-La Mancha con Facultad de Educación. De estas 1.623 personas iniciales, el estudio se ha realizado sobre 1.308 de ellas, aquellas que presentaron sus cuestionarios de recogida de información perfectamente cumplimentado. Del total de 1.308, 354 son hombres (un 27\%), y 954, mujeres (un 73\%). La edad media es de 26,23 años, y el rango de edad, de 18 a 56 años. 
Tabla 1. Distribución de la muestra por ciudades

\begin{tabular}{lcc}
\hline & Total & Válidos \\
\hline Ciudad Real & 549 & 429 \\
Cuenca & 306 & 279 \\
\hline Toledo & 462 & 348 \\
\hline Albacete & 303 & 252 \\
\hline Total & 1.623 & 1.308 \\
\hline
\end{tabular}

Fuente: elaboración propia.

\subsection{Procedimiento}

La información se ha recogido en horario lectivo. Con las diferentes facultades de Educación, se ha establecido un protocolo para la administración de las pruebas y las recogidas de la información, donde se determinan: fechas de recogida y envío de las pruebas, instrucciones para cumplimentar el cuestionario, declaración de privacidad y realización voluntaria del cuestionario.

Una vez recogida la información, se han seleccionado los cuestionarios válidos, se ha construido la base de datos y se han establecido las variables necesarias para la realización de los cálculos esenciales. Además de comprobar la fiabilidad y la estructura de la prueba, se han realizado análisis estadísticos y descriptivos para conocer los valores de nuestra muestra, así como varios test de comparación de medias (prueba $t$ para muestras independientes), a fin de conocer las significatividad de las puntuaciones obtenidas por hombres y mujeres en los diferentes valores de sexismo. Hemos calculado la correlación entre las diferentes medidas de sexismo obtenidas (prueba de correlación bivariada).

Para el desarrollo de los cálculos sobre sexismo, el punto de corte en las subescalas de sexismo hostil y de sexismo benévolo se ha colocado en el primer cuartil (11 para sexismo hostil y 13 para sexismo benévolo).

\section{Instrumento de recogida de información}

Para el desarrollo del proceso de recogida de información, se ha construido un test en el que se incluye una primera parte destinada a recoger datos sociodemográficos de cada participante y una segunda parte destinada a recopilar datos referidos a la actitud sexista de cada participante.

- Variables sociodemográficas: edad, sexo, ciudad, titulación.

- Inventario de sexismo ambivalente (ASI) (Expósito, Moya y Glick, 1998): adaptación y validación al castellano de la prueba original de Glick y Fiske (1996) que mide los dos tipos de sexismo (hostil y benévolo). Se trata de una escala tipo Likert con puntuaciones que van desde 0 (totalmente en desacuerdo) hasta 5 (totalmente de acuerdo), donde, a mayor puntuación, mayores creencias sexistas. Los ítems 1, 3, 6, 8, 9, 12, 13, 17, 19, 20 y 22 nos dan la medida de sexismo benévolo y los ítems 2, 4, 
$5,7,10,11,14,15,16,18$ y 21 ofrecen como resultado la medida en sexismo hostil. En cuanto a la fiabilidad, hemos obtenido un coeficiente Alfa de 0,92 (0,90 en la subescala de sexismo hostil y 0,86 en la subescala de sexismo benévolo).

\section{Resultados}

Presentamos los resultados obtenidos en nuestro trabajo de investigación ordenado en torno a nuestras hipótesis de trabajo.

Los estudiantes de Magisterio puntúan más alto en sexismo hostil y en sexismo benévolo que las estudiantes de Magisterio.

Tabla 2. Diferencias entre hombres y mujeres. Prueba $t$ de Student

\begin{tabular}{lcc}
\hline & Sexismo hostil & Sexismo benévolo \\
\hline Mujer & 17,25 & 20,31 \\
\hline Hombre & 26,44 & 25,37 \\
Sig. bilateral & $\mathbf{0 , 0 0}$ & $\mathbf{0 , 0 0}$ \\
\hline
\end{tabular}

Fuente: elaboración propia.

Los resultados muestran que los hombres puntúan significativamente más alto que las mujeres en sexismo hostil y en sexismo benévolo.

Tabla 3. Correlación entre sexismo hostil y sexismo benévolo

\begin{tabular}{llc}
\hline & & Sexismo benévolo \\
\hline \multirow{2}{*}{ Sexismo hostil } & Correlación de Pearson & 0,76 \\
& Sig. bilateral & 0,00 \\
\hline
\end{tabular}

Fuente: elaboración propia.

Desde una perspectiva estadística, los resultados muestran una correlación muy significativa entre sexismo hostil y sexismo benévolo.

Los hombres puntuan significativamente más alto en sexismo hostil que en sexismo benévolo. 
Tabla 4. Medias en sexismo de hombres. Prueba $t$ de Student

\begin{tabular}{lccc}
\hline Hombre & Sexismo hostil & Sexismo benévolo & Sig. bilateral \\
\hline Puntuación & 26,44 & 25,37 & 0,00 \\
$N$ & 330 & 306 & \\
\hline Porcentaje & $93,22 \%$ & $86,4 \%$ & \\
\hline
\end{tabular}

Fuente: elaboración propia.

Los resultados muestran que los hombres puntúan significativamente más alto en sexismo hostil que en sexismo benévolo y que hay más hombres que puntúan alto en sexismo hostil que en sexismo benévolo.

Las mujeres puntúan significativamente más alto en sexismo benévolo que en sexismo hostil.

Tabla 5. Medias en sexismo del grupo de las mujeres. Prueba $t$ de Student

\begin{tabular}{lccc}
\hline \multicolumn{1}{c}{ Mujer } & Sexismo hostil & Sexismo benévolo & Sig. bilateral \\
\hline Puntuación & 17,25 & 20,31 & 0,00 \\
$N$ & 675 & 666 & \\
Porcentaje & $70,75 \%$ & $69,8 \%$ & \\
\hline
\end{tabular}

Fuente: elaboración propia.

Los resultados indican que las mujeres puntúan significativamente más alto en sexismo benévolo que en sexismo hostil. Sin embargo, en nuestra muestra, hay más mujeres con creencias sexistas hostiles que benévolas.

Más del $51 \%$ de los estudiantes y las estudiantes de Magisterio presentan creencias sexistas hostiles y benévolas.

Tabla 6. Porcentaje de la población con creencias sexistas

\begin{tabular}{lcc}
\hline Población & Sexismo hostil & Sexismo benévolo \\
\hline$N: 1.308$ & 996 & 987 \\
Porcentaje sexista & 76,14 & 75,55 \\
\hline
\end{tabular}

Fuente: elaboración propia.

Los resultados muestran que el 76,14 de la población estudiada puntúa alto en sexismo hostil y el $75,55 \%$ de esta puntúa alto en sexismo benévolo. 


\section{Análisis y discusión de los resultados}

Tal como también hicimos con el bloque de resultados, para desarrollar este bloque de contenidos, hemos decidido estructurarlo en función de nuestras hipótesis de trabajo.

\subsection{Los estudiantes de Magisterio puntúan más alto en sexismo hostil $y$ en sexismo benévolo que las estudiantes de Magisterio}

Los resultados de nuestro trabajo confirman esta hipótesis de trabajo, dado que los hombres puntúan significativamente más alto que las mujeres en sexismo benévolo y en sexismo hostil, sobre todo en sexismo hostil. Estos resultados están alineados con los encontrados en investigaciones anteriores (Carretero, 2011) y en investigaciones desarrolladas con otras poblaciones (Cárdenas, Lay, González, Calderón y Alegría, 2010).

Los resultados muestran, también, como, a pesar de estas diferencias, hombres y mujeres obtienen una puntuación media en sexismo hostil y en sexismo benévolo consideradas como sexistas, dado que, en todos los casos, sus puntuaciones superan el punto de corte por encima del cual se considera que la puntuación obtenida es sexista (hostil y benévola).

Las pruebas de correlación bivariada muestran una relación importante (correlación de Pearson 0,68) y una significatividad de esta correlación (sig. bilateral 0,00), lo que implica que, en nuestra muestra, ambas medidas en sexismo están relacionadas. Dichos resultados coinciden con los encontrados con anterioridad (Carretero, 2011), así como los hallados en investigaciones sobre sexismo en otras poblaciones de estudio (López-Cepero, RodríguezFranco, Rodríguez-Díaz y Bringas, 2013).

Nos llama la atención, y es algo que no esperábamos encontrar, que hombres que han elegido estudiar una titulación tradicionalmente escogida por mujeres, donde se desarrolla un rol —el de educador cuidador- atribuido al mundo femenino desde la ideología de género tradicional, presenten actitudes sexistas, lo que de alguna manera contradice sus propias creencias. Nos preguntamos acerca de los motivos de su elección.

Estos resultados no solo parecen mostrar las diferencias entre hombres y mujeres respecto a creencias sexistas, sino también las probables diferencias en las prácticas educativas que desarrollarán ellos y ellas según los resultados encontrados, donde la probabilidad de que el docente trasmita la ideología sexista e implemente procesos educativos sexistas es sensiblemente más alta si el docente es un hombre.

\subsection{Los hombres puntúan significativamente más alto en sexismo hostil que en sexismo benévolo}

Los resultados encontrados en nuestro análisis confirman nuestra hipótesis de trabajo y muestran cómo los hombres que han participado en él presentan 
creencias sexistas hostiles y benévolas con puntuaciones en ambos casos por encima del punto de corte, siendo, además, su puntuación en sexismo hostil significativamente más alta que en sexismo benévolo. Dichos resultados confirman los encontrados con anterioridad en estudios con muestras diferentes (Moya y Expósito, 2001; Lameiras y Rodríguez, 2004).

Además, más del $92 \%$ de los hombres que han participado en nuestro estudio presenta estas creencias, frente al $86 \%$ que lo hacen en sexismo benévolo. Es decir, son más los hombres que puntúan alto en sexismo hostil, y además la puntuación en sexismo hostil es significativamente más elevada que en sexismo benévolo. Estos hombres afrontarán su tarea como profesores, así como sus relaciones con el resto de compañeros, compañeras y con su institución educativa, desde esta actitud sexista, que, por tanto, puede manifestarse en todos y cada uno de los contextos y de los procesos en los que el docente participe: clases, coordinación y reuniones con madres y padres, órganos de gobierno, consejos, claustros, etc.

Según estos resultados, prácticamente la totalidad de los futuros hombres enseñantes presenta, con mayor o menor intensidad, actitudes sexistas hostiles, lo que significa que, llegado el momento de ejercer la docencia, lo harán desde esta posición, con las implicaciones que pueda comportar su actitud: reparto de roles, de tareas, de desarrollo de capacidades y de oferta de oportunidades diferentes para niñas y niños.

No podemos pasar por alto la puntuación en sexismo benévolo, que, si bien es cierto que se produce en menos hombres y con menor intensidad, no por ello es inexistente.

\subsection{Las mujeres puntúan significativamente más alto en sexismo benévolo que en sexismo hostil}

Los resultados encontrados confirman nuestra hipótesis de trabajo. Las mujeres, tal como ocurría con el grupo de los hombres, puntúan por encima del punto de corte en sexismo hostil y benévolo, por lo que presentan actitudes sexistas. En su caso, la puntuación obtenida en sexismo benévolo es significativamente más alta que la de sexismo hostil. Estos resultados están en la línea de los encontrados en estudios y en trabajos anteriores (Rodríguez, Lameiras, Carrera y Faílde, 2009). En la muestra que hemos obtenido, hay más mujeres con actitud hostil que benévola, aunque la puntuación es más elevada en sexismo benévolo.

En nuestro trabajo, las mujeres no solo puntúan significativamente de modo más bajo en sexismo hostil y benévolo que los hombres, sino que, además, en ambas dimensiones, su puntuación es sensiblemente menor.

Puede significar que, como grupo, las mujeres que han tomado parte en esta investigación presenten una posición ideológica significativamente diferente a la de los hombres. Sin embargo, no podemos obviar que en torno al $70 \%$ de las mujeres de nuestra población de objeto de estudio presenta actitudes sexistas, lo que implica que la probabilidad de trasmisión del sexismo a 
través de la práctica educativa en el caso de que el docente sea mujer es también muy elevada, especialmente por lo que se refiere al componente benévolo del sexismo.

\subsection{Más del $51 \%$ de los estudiantes y de las estudiantes de Magisterio presentan creencias sexistas hostiles y benévolas}

En nuestro trabajo, más del $75 \%$ de la población objeto de estudio ha puntuado por encima de la línea de corte en sexismo benévolo y en sexismo hostil. Estos resultados mantienen y confirman nuestra hipótesis de trabajo.

Son porcentajes especialmente altos los encontrados en el colectivo de los hombres, lo que, insistimos, creemos que entra en contradicción con la elección formativa y laboral que han realizado para su futuro.

Como ya hemos mencionado, los hombres puntúan más alto en ambos tipos de sexismo y son más los que presentan actitudes sexistas que las mujeres. Sin embargo, también el grupo femenino muestra dichas actitudes.

Estos resultados indican la alta dificultad de desarrollar y de implementar los objetivos y los contenidos incluidos en la legislación educativa vigente referidos a la igualdad y a la no discriminación, puesto que el futuro docente parte de una posición ideológica y actitudinal incompatible con dichos objetivos y contenidos.

En realidad, de confirmar estos resultados con una muestra más amplia de estudiantes y con el colectivo de docentes en activo, nos encontraríamos con una situación legal que no sería posible llevar a la práctica, dada, insistimos, la ideología y la actitud sexista de base existente en la gran mayoría de personas responsables de la educación.

Los resultados encontrados, por sí mismos, indican la necesidad de incluir contenidos sobre género, sexismo y perspectiva de género en la formación necesaria para ejercer la docencia.

\section{Conclusiones}

Los resultados encontrados en nuestro estudio muestran una población de futuros docentes con actitudes sexistas en más de un 75\% de los casos. Esta actitud está presente en un mayor porcentaje de hombres y con mayor intensidad también en el caso masculino. Sin embargo, tanto ellos como ellas, como grupo, presentan creencias y actitudes sexistas. Por sí mismos, estos resultados indican la necesidad de actuar, desde nuestra perspectiva, en varios niveles: en primer lugar, consideramos la urgencia de diseñar los planes de estudio de las facultades de Educación desde una perspectiva de género, donde conceptos como igualdad y no discriminación sean centrales, y no solo descriptivos; en segundo lugar, consideramos necesaria la inclusión de objetivos y de contenidos en forma de créditos obligatorios relativos al género, el sexismo y su relevancia en los procesos educativos; en tercer lugar, creemos en la enorme utilidad de programar acciones formativas vinculadas a las facultades de 
Educación en relación con el trabajo personal sobre sistemas de creencias, modificación de actitudes y cambio de perspectiva en torno a los modelos tradicionales y al sexismo como principal actitud de discriminación hacia el universo femenino, $y$, en cuarto lugar, entendemos que la legislación relativa a la igualdad de género no puede ser desarrollada desde posiciones y actitudes sexistas que contradigan lo que se pretende legislar, por lo que estas normas, o las partes relacionadas con estos contenidos, pueden ser desarrolladas o contar con el asesoramiento de profesionales que partan desde posiciones alineadas con lo que se pretende legislar.

Los resultados encontrados muestran una población de estudiantes que aspira a ejercer la docencia que mantiene las mismas creencias que el resto de la población. Si la educación tiene que ser el motor de cambio y de desarrollo de nuestra sociedad, es necesario que sus profesionales sean capaces de implementar y desarrollar esas modificaciones que se pretenden conseguir en la sociedad en ellos mismos, porque, si no es así, puede que estemos abocados a repetir y perpetuar el mismo modelo social en lo fundamental y que los cambios se realicen en aspectos más superficiales o menos fundamentales. Las modificaciones en herramientas de trabajo, en medios de actuación o en organización de la clase son más o menos simples de alcanzar. Los cambios a nivel ideológico y cultural son más complejos de conseguir, puesto que necesitan de la trasformación de mentalidad por parte de los profesionales implicados, y no solo del contexto o la norma. Por otro lado, los resultados obtenidos explican, al menos en parte, la tasa tan elevada de matriculación de mujeres en los estudios de Magisterio (más del 70\%).

Tal como esperábamos, los resultados presentan al grupo de las mujeres con puntuaciones significativamente más altas en sexismo benévolo. Si tenemos en cuenta que el número de matrículas femeninas, frente al de masculinas, en los estudios de Magisterio está en el 73\%, en el caso de nuestro estudio, nos encontramos con que esta forma benévola del sexismo será la que tendrá más presencia en nuestras aulas en el futuro.

Nos preguntamos por qué hombres que puntúan alto en las dos dimensiones de sexismo se matriculan en unos estudios que consideran propios de mujeres. En la escuela actual, aún son más los hombres que ocupan puestos directivos, con mayores obligaciones de gestión, administración y dirección y menos obligaciones y carga en horas lectivas, y esta puede ser una de las posibles explicaciones a este hecho. Otra explicación podría estar relacionada con aspectos relativos a las notas de cada uno y a las posibilidades que asignan esas notas en la elección de estudios (podríamos buscar cuántos hombres con notas superiores al 8,5 se inclinan por estudiar Magisterio). También pueden influir en esta elección las expectativas acerca de las condiciones laborales propias del puesto de maestro.

Por último, y relacionado exclusivamente con nuestras creencias, parece que la población de estudiantes de Magisterio no difiere, en cuanto a creencias sexistas, de otras poblaciones de estudiantes de otras titulaciones o de la población en general, al contrario de lo que esperábamos encontrar. 


\section{Limitaciones de la presente investigación}

La presente investigación ha sido realizada con estudiantes de la Comunidad de Castilla-La Mancha y consideramos que, aunque se trate de una muestra representativa de ellos, no podemos, sin embargo, generalizar sus resultados a la población de docentes en activo, por lo que las conclusiones hablan de posibilidades de futuro, no de lo que pueda o no estar ocurriendo actualmente.

Consideramos que las variables sociodemográficas podrían aportar mucha más información si, en análisis sucesivos, incluyéramos el estudio de otras variables como el lugar de procedencia de cada participante, la religión (considerando la legislación vigente), el estilo educativo de la familia de origen y las actitudes de los componentes de la familia de origen, con objeto de encontrar y concretar más las raíces y la causa de las actitudes que aparecen en este estudio y poder así concretar también las medidas a tomar y la población objeto de esas medidas.

La presente investigación de corte cuantitativo nos permite conocer la generalidad, el estereotipo de la población estudiada, y esta información es importante para diseñar acciones globales y/o generales. Sin embargo, para el desarrollo de medidas locales, concretas, consideramos necesario que se implementen procesos cualitativos que nos permitan conocer más en concreto la población objeto de las acciones que se decidan emprender. Esto supone una inversión económica y temporal importante, a la vez que puede aumentar las probabilidades de éxito de las acciones a desarrollar.

\section{Líneas de trabajo abiertas}

En el futuro inmediato, planteamos la necesidad de implementar investigaciones con la finalidad de confirmar, o no, estos resultados con la población de estudiantes de Magisterio de todo el país, a fin de establecer, no solo las medidas a tomar, sino también el ámbito de actuación y las posibles diferencias según las diversas zonas estudiadas. Al mismo tiempo, nos parece fundamental conocer cuáles son las creencias y las actitudes sexistas de la población docente activa, con objeto de plantear posibles medidas de actuación también con esta población, y no solo con los docentes.

En cuanto a los estudiantes de Magisterio de Castilla-La Mancha, consideramos la necesidad de replantear el contenido de los planes de estudio y su diseño, así como la inclusión de la formación obligatoria, complementaria, optativa y/o de libre configuración que se oferte desde las propias facultades o desde los diferentes campus universitarios y recursos educativos. El diseño de la formación en cuanto a objetivos, contenidos, metodología y metas debe adecuarse al contexto en el que se desarrollará, pero sin perder de vista la perspectiva de género ni la consecución de la igualdad entre los dos sexos. En concreto, consideramos la inclusión de créditos obligatorios acerca del género, el sexismo, la igualdad y la perspectiva de género que facilite al alumnado la clarificación de conceptos y la comprensión de los procesos que generan y que 
forman cada uno de esos conceptos, así como la manera en que se relacionan unos con otros y las consecuencias surgidas en relación con la educación. Al mismo tiempo, proponemos la programación de cursos y de seminarios donde se profundice en la toma de conciencia y en el reconocimiento de las propias ideologías de género, en las actitudes sexistas y su utilidad, en las alternativas y en los costes como manera de modificar y de facilitar el cambio ideológico y actitudinal y formar docentes capaces de trabajar por y para la igualdad desde lo que son, y no solo desde lo que dice la norma.

Valoramos la posibilidad de desarrollar una prueba que permita recoger información acerca del sexismo de una manera diferente, centrándose en la ejecución y no solo en la información que cada participante facilite, con los sesgos que este tipo de pruebas implique en su desarrollo.

\section{Referencias bibliográficas}

Ayres, M.M.; Friedman, C.K. y Leaper, C. (2009). Individual and situational factors related to young women's likelihood of confronting sexism in their everyday lives. Sex Roles, 61, 441-460.

Barreto, M.; Ellemers, N.; Cihangir, S. y Stroebbe, K. (2009a). The self-fulfilling effects of contemporary sexism: How it affects women's well-being and behavior. En M. Barreto, M. Ryan y M. Schmitt (eds.). The glass ceiling in the 21st century: Understanding barriers to gender equality (pp. 99-123). Washington, DC: American Psychological Association.

Barreto, M.; Ryan, M.K. y Schmitt, M.T. (2009b). The glass ceiling in the 21st century: Understanding barriers to gender equality. Washington, DC: American Psychological Association.

Blakemore, J.O.; Berenbaum, S.A. y Liben, L.S. (2009). Gender Development. Nueva York: Psychology Press.

Cárdenas, M.; LaY, S.-L.; González, C.; Calderón, C. y Alegrría, I. (2010). Inventario de sexismo ambivalente: Adaptación, validación y relación con variables psicosociales. Salud y Sociedad, 2(1), 125-127.

Carretero, R. (2011). Sexo, sexismo y acoso escolar entre iguales. Complutense Educación, 22(1), 27-43.

Díaz-Aguado, M.J. (2006). Sexismo, violencia de género y acoso escolar. Revista de Estudios de la Juventud, 73, 38-57.

Díaz-Aguado, M.J.; Martínez, R. y Martín, J. (2010). Estudio sobre la convivencia escolar en la educación secundaria obligatoria. Madrid: Ministerio de Educación.

Eagly, A. y Sczesny, S. (2009). Stereotypes about women, men, and leaders: Have times changed? En M. Barreto, M.K. Ryan y M.T. Schmitt (eds.). The glass ceiling in the 21st century: Understanding barriers to gender equality (pp. 21-47). Washington, DC: American Psychological Association.

Expósito, F. y Herrera, M.C. (2009). Social percepction of violence against women: Individual and psychosocial characteristics of victims and abusers. European Journal of Psychology Applied to the Legal Context, 1(1), 123-145.

Expósito, F. y Moya, M. (2005). Violencia de género. En F. Expósito y M. Moya (eds.). Aplicando la psicología social (pp. 2001-227). Madrid: Pirámide.

Expósito, F.; Moya, M. y GLick, P. (1998). Sexismo ambivalente: Medición y correlatos. Revista de Psicología Social, 13(2), 159-169. 
Ferragut, M.; Blanca, M.J. y Ortiz-Tallo, M. (2013). Psychological values as protective factors against sexist attitudes in preadolescents. Psicothema, 25(1), $38-42$.

Garaigordobil, M. (2013). Sexism and alexithymia: Correlations and differences as a function of gender, age, and educational level. Anales de Psicología, 29(2), 368-377.

Garaigordobil, M. y Aliri, J. (2013). Relaciones del sexismo con justificación de violencia y con otras formas de prejuicio como la dominancia social y el autoritarismo. Estudios de Psicología, 34(2), 127-139.

Gartzia, L. (2011). Nuevos modelos de gestión: De pensar en masculino a pensar en andrógino. Boletín de Estudios Económicos, 203, 299-314.

Gartzia, L.; Aritzeta, A. y Balluerka, N. (2012). Inteligencia emocional y género: Más allá de las diferencias sexuales. Introducción. Anales de Psicología, 28, 567-575.

GLick, P. y Fiske, S.T. (1996). The ambivalent sexism inventory: Differentiating and Hostil Benevolent sexism. Journal of Personality and Social Psychology, 70, 491-512.

- (1999). Hostil and benevolent sexism: Measuring ambivalent sexist attitudes. Psychology of Women Quarterly, 21, 119-135.

Gorostiaga, A.; Balluerka, N. y Soroa, G. (2014). Evaluación de la empatía en el ámbito educativo y su relación con la inteligencia emocional. Revista de Educación, 364, 43-61.

Lameiras, M. (2004). El sexismo y sus caras: De la hostilidad a la ambivalencia. Anuario de Sexología, 8, 91-102.

Lameiras, M.; López, W.; Rodríguez, Y.; Ávila, M. d'; Lugo, I.; Salvador, C. y Granejo, M. (2002). La ideología de rol sexual en Iberoamérica. Avances en Psicología Clinica Latinoamericana, 20, 37-44.

Lameiras, M. y Rodríguez, A. (2004). Evolution of hostil sexism and benevolent sexism in a Spanish sample. Social Indicators Research, 66, 197-201.

LEA, H. (2011). The role of gender, affectivity and parenting in the course of eating disorders: A 4-year prospective case-control study among adolescents. International Journal of Nursing Studies, 48, 959-972.

Ley Orgánica 2/2006, de 3 de mayo, de educación (LOE). Boletín Oficial del Estado, 106 (4 mayo 2006).

Ley Orgánica 8/2013, de 9 de diciembre, para la mejora de la calidad educativa. Boletin Oficial del Estado, 295 (10 diciembre 2013).

López-Cepero, J.; Rodríguez-Franco, L.; Rodríguez-Díaz, F. y Bringas, C. (2013). Validación de la versión corta del Social Roles Questionnaire (SRQ-R) con una muestra adolescente y juvenil española. Revista Electrónica de Metodología Aplicada, 18(1), 1-16.

Merino, E.; Martínez, M. y Díaz-Aguado, M.J. (2010). Sexismo, inteligencia emocional y adolescencia. Revista de Psicología Educativa, 16(1), 77-88.

Moya, M. (2004). Actitudes sexistas y nuevas formas de sexismo. En E. Barbera e I. Martínez-Benlloch (eds.). Psicología y Género (pp. 271-294). Madrid: Pearson.

- (2006). Revisión de las propiedades psicométricas de las versiones larga y reducida de la Escala sobre Ideología de Género 1. International Journal of Clinical and Health Psychology, 6, 709-727.

Moya, M. y Expósito, F. (2001). Nuevas formas, viejos intereses: Neosexismo en varones españoles. Psicothema, 13, 668-674.

Moya, M.; Glick, P.; Expósito, F; Lemus, S. de y Hart, J. (2007). Its for your own good: Benevolent sexism and women's reactions to proetectively justified restrictions. Personality and Social Psychology Bulletin, 33(10), 1421-1434. 
Ovejero, A.; Yubero, S.; Larrañaga, E. y Navarro, R. (2013). Sexismo y comportamiento de acoso escolar en adolescentes. Psicología Conductual, 21(1), 157-171.

Rodríguez, M.J.; Lameiras, M.; Carrera, M.V. y Faílde, J.M. (2009). Aproximación conceptual al sexismo ambivalente: Estado de la cuestión. SUMMA Psicológica UST, 6(2), 131-142.

Roets, A.; Van Hiel, A. y Dhont, K. (2012). Is sexism a gender issue?: A motivated social cognition perspective on men's and women's sexist attitudes toward the own and other gender. European Journal of Personality, 26, 350-359.

Rudman, L.A. y Glick, P. (2001). Prescriptive gender stereotypes and backlash toward agentic women. Journal of Social Issues, 57, 743-762.

VAAmONDE, J.D. (2010). Valores y sexismo en adolescentes argentinos. Revista Salud y Sociedad, 1, 113-124.

Zakrisson, I.; Aderzén, M.; Lenell, F. y Sandelin, H. (2012). Ambivalent sexism: A tool for understanding and improving gender relations in organizations. Scandinavian Journal of Psychology, 53, 64-70. 